\title{
Analysis of the SMOS Ocean Salinity Inversion Algorithm
}

\author{
Carolina Gabarró*, Marcos Portabella ${ }^{\dagger}$, Marco Talone ${ }^{\ddagger}$ and Jordi Font* \\ *Institut de Ciències del Mar de Barcelona (CMIMA-CSIC) \\ Barcelona 08003, Spain. E-mail: cgabarro@icm.csic.es \\ ${ }^{\dagger}$ Koninklijk Nederlands Meteorologisch Instituut (KNMI). \\ Postbus 201, 3730 AE de Bilt. The Netherlands \\ $\ddagger$ Departament Teoria del Senyal i Comunicacions (TSC) \\ Universitat Politècnica de Catalunya (UPC). Barcelona, Spain
}

\begin{abstract}
As part of the preparation for the European Space Agency SMOS (Soil Moisture and Ocean Salinity) satellite mission, empirical sea surface emissivity (forward) models have been applied to retrieve sea surface salinity from L-band brightness temperature $\left(T_{B}\right)$ measurements. However, the salinity inversion is not straightforward and an important effort is required to define the most appropriate cost function (inversion algorithm).

Different Bayesian-based configurations of the cost function are examined, depending on whether prior information is used in the inversion or not. It is important to properly balance all the terms of the cost function, as well as to have a good knowledge of the quality of the prior information. A sensitivity analysis shows that the instrument has low sensitivity to the geophysical parameters that modulate the Tb (including salinity). As such, the inversion needs to be constrained with prior information. Simulations are also performed using the SMOS simulator to assess the retrieval errors produced by the different cost function configurations. In line with the sensitivity analysis, the errors are very large when no prior information is used in the cost function. The lowest errors are obtained when the inversion is constrained with the full prior information, i.e., information from all the auxiliary (geophysical) parameters. As such, it is concluded that the use of prior information is essential for a successful salinity retrieval from SMOS measurements.
\end{abstract}

\section{SALINITY DETERMINATION BY L-BAND RADIOMETRY}

The knowledge of the distribution of the sea surface salinity (SSS) at a global scale and its inter-annual variability is vital to understand the ocean role in the Earth climate. The European Space Agency's SMOS (Soil Moisture and Ocean Salinity) mission, due for launch in mid 2008, aims at generating global SSS maps with a spatial and temporal resolution adequate for climate and ocean general circulation studies. For such purpose, an L-band interferometric radiometer with full polarimetric capability called MIRAS (Microwave Imaging Radiometer by Aperture Synthesis) will be the SMOS payload. This instrument will provide at each overpass a 2D image of the ocean surface brightness temperature, observing the same point under a wide range of incidence angles, which is crucial for a successful salinity retrieval.

The forward model or geophysical model function (GMF) used in this analysis is the Klein and Swift model [1] for the flat sea and the Hollinger model [2] for the roughness contribution, described by the wind speed parameter (WS) and the incidence angle $(\theta)$ as follows:

$$
\begin{aligned}
& T b_{h}=0.2 *(1+\theta / 55) W S \\
& T b_{v}=0.2 *(1-\theta / 55) W S
\end{aligned}
$$

The GMF therefore assumes that L-band brightness temperature measurements of the ocean surface are modulated by the sea surface temperature (SST) and sea surface salinity (SSS) (i.e., flat sea contribution) and the sea surface wind speed (WS) for the roughness contribution.

The inversion is not straightforward since the GMF is non-linear (flat sea term) and there are three geophysical parameters to derive (SSS, SST and WS).

A Bayesian approach is used to perform the salinity inversion. Assuming that measurement errors are Gaussian and uncorrelated, a quadratic inversion cost function, which allows to locally invert salinity, is formulated. A suitable definition of the Bayesian-based cost function is very important to obtain a good performance in the salinity retrieval process from SMOS measurements. In this paper, several cost functions are formulated and tested with simulated data.

\section{COST FUNCTION DEFINITION}

Three different Bayesian-based cost functions have been considered: the first one is the case where no prior information is used, i.e., the cost function consists of an observation term where all the parameters are 'free' in the minimization procedure; the second one is the case for which prior (background) information together with proper characterization of both the measurement and the background errors are also introduced in the cost function. Finally, a hybrid configuration considering prior information on the auxiliary parameters, but not on SSS is also considered.

When no constraints (prior information) are considered, the cost function is defined as follows:

$$
\chi^{2}=\frac{1}{N} \sum_{i=0}^{N-1} \frac{\left[T_{B_{i}}^{\text {meas }}-T_{B_{i}}^{\text {model }}\left(\theta_{i}, S S S, S S T, \text { Rough }\right)\right]^{2}}{\sigma_{T_{B}}^{2}}
$$


where $\theta_{i}$ is the incidence angles and $\mathrm{N}$ is the number of different observations, $T_{B_{i}}^{\text {meas }}$ is the measured brightness temperature, and $T_{B_{i}}^{\text {model }}$ is the modelled brightness (obtained through the forward model).

On the other hand, when constraints are considered the cost function is defined as follows:

$$
\begin{array}{r}
\chi^{2}=\frac{1}{N} \sum_{i=0}^{N-1} \frac{\left[T_{B_{i}}^{\text {meas }}-T_{B_{i}}^{\text {model }}\left(\theta_{i}, \text { SSS SSST }, \text { Rough }\right)\right]^{2}}{\sigma_{T_{B}}^{2}} \\
+\sum_{j} \frac{\left[P_{j}-P_{j \text { prior }}\right]^{2}}{\sigma_{P_{j}}^{2}}
\end{array}
$$

where $P$ is the geophysical parameter to be found, with $j$ possible parameters (i.e.: SSS, SST and WS in our case), $P_{\text {prior }}$ is a reference value (or prior), for each of the parameters (obtained from satellite or model outputs) from which the final solution should not be far, and $\sigma_{P}^{2}$ is the variance of the expected error of the reference values. The value of $P$ at the first iteration is the so-called "first guess" value. Then the $99 \%$ of the solutions of $P$ will range from $P_{\text {prior }}-3 * \sigma_{P}$ to $P_{\text {prior }}+3 * \sigma_{P}$. If a reference value of the parameters is known with low precision, $\sigma_{P}$ is large, then the term of this parameter is small, and has less weight in the overall equation.

The third case, the so-called hybrid case, is also formulated as in 3 but $\sigma_{S S S}$ is set to a very large value to make this term null.

\section{SENSITIVITy ANALYSIS}

To get an idea of the instrument sensitivities and their impact in the different cost function formulations, a visualization of several cost function cuts (planes) has been carried out for a pre-defined (simulated) case. The original parameters (truth) are set to $S S S_{\text {orig }}=35 \mathrm{psu}, W S_{\text {orig }}=5 \mathrm{~m} / \mathrm{s}$ and $S S T_{\text {orig }}$ $=20{ }^{\circ} \mathrm{C}$. The GMF defined in section I is used to simulate the $\mathrm{Tb}$ values for 55 different incidence angles, from $0^{\circ}$ to $55^{\circ}$. Afterwards a Normal Distribution noise with standard deviation of $2 \mathrm{~K}$ has been added to the $T_{B_{\text {orig }}}$ generated, that ranges in $T_{B h}$ from 94 to $60 \mathrm{~K}$ and in $T_{B v}$ from 94 to 140 $\mathrm{K}$.

In the case of using constraints, the references used are as follows: $S S S_{\text {ref }}=S S S_{\text {orig }}+1 \mathrm{psu}, W S_{\text {ref }}=W S_{\text {orig }}+1.5$ $\mathrm{m} / \mathrm{s}, S S T_{\text {ref }}=S S T_{\text {orig }}+0.25^{\circ} \mathrm{C}$, and the uncertainties of these references are set to $\sigma_{S S S}=2 \mathrm{psu}, \sigma_{W S}=2.5 \mathrm{~m} / \mathrm{s}$, $\sigma_{S S T}=0.5^{\circ} \mathrm{C}$.

Contour plots have been created for several configurations of the cost function which show the behavior of the minima in 2-D (a 2-D cut in a 3-D cost function), and indicate if more than one minimum is present, and if the minima are well defined or broad.

Figure 1 plots the cost function behavior when varying SSS and WS, while SST has been set to the original value. They show the cases when no constraints (plot a), only one (plots b, c), or contrains in all parameters are used (plot d). They present for all the cases a unique minimum and are located at the following positions: for case a) in $\mathrm{SSSmin}=35.10$ psu and WSmin $=5.10 \mathrm{~m} / \mathrm{s}$, for case b) $\mathrm{SSSmin}=35.80 \mathrm{psu}$

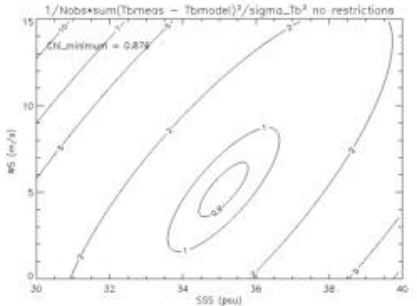

a)

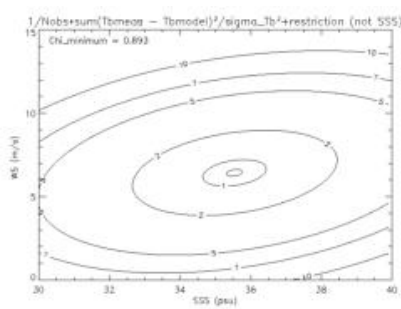

c)

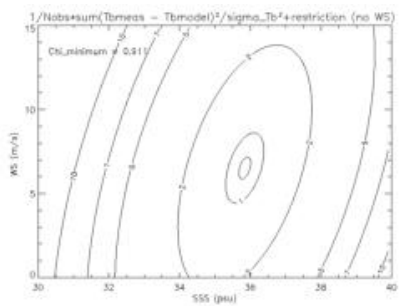

b)

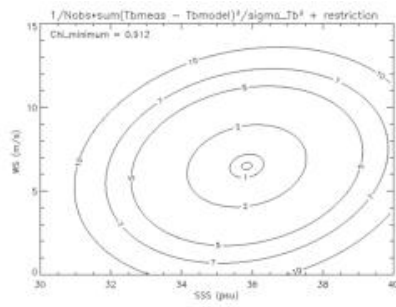

d)
Figure 1. Cost function value $\left(\chi^{2}\right)$ contour when varying SSS and WS parameters for (a) no constraints, (b) with SSS constraints only, (c) with WS constraints only and (d) when all constraints are used.

and WSmin $=6.45 \mathrm{~m} / \mathrm{s}$, for case c) SSSmin $=35.50 \mathrm{psu}$ and WSmin $=6.45 \mathrm{~m} / \mathrm{s}$ and for case d) SSSmin $=35.80 \mathrm{psu}$ and WSmin $=6.45 \mathrm{~m} / \mathrm{s}$.

Plot 1a shows the similar sensitivity of $T_{B}$ to SSS and WS, as indicated by the broad minimum oriented approximately along the diagonal of the plot. By increasing or decreasing both WS and SSS along the major axis of the elliptic contours, similar cost function values (i.e., similar $T_{B}$ values) are obtained for a wide range of SSS and WS. This indicates the low sensitivity of the instrument to both SSS and WS changes.

An indirect but straightforward way to quantify the sensitivity is to compare the weight of the minimum with the weight of to the cost function points in the vicinity of the minimum. That is, the probability that a cost function point corresponds to the true solution can be theoretically calculated by the following expression: $P=\exp \left(-\chi^{2} / 2\right)$, where $\chi^{2}$ is the Maximum Likelihood Estimation [3]. In the case of no constraints (a) the probability to fall into the minimum is very similar to the probability to be over the contour line of $\chi^{2}=1$, which has a quite extensive range of possible solutions (SSS from 33.5 to $37.5 \mathrm{psu}$ and WS from 2 to $9 \mathrm{~m} / \mathrm{s}$ ). The probability to be over the contour line of $\chi^{2}=2$ is half of the probability to find the minimum, and this line includes almost all the realistic possible solutions. This means that the sensibility to SSS is very low and that it is very likely to converge to a solution which is not the real minimum. Therefore, taking into account that the GMF sensitivities are realistic, it is clear that a cost function without constraints will lead to large retrieval errors when real (noisy) $T_{B}$ are used as input.

When only 1 parameter is restricted (plots b and c), the range of solutions with meaningful probability is reduced with respect to no constraints (plot a), but still considerable. 
Figure 1d shows that if all the constraints are used the minimum is well defined. However, the retrieved parameters tend to the reference values. This was expected since it has been shown that the observational terms give little information, but it can be optimized by balancing the observational and the background terms such that the information content of the measurements is fully exploited. For such purpose, proper characterization of both the measurement and background uncertainties is crucial, and should be done once the satellite will fly and real data will be acquired (during the commissioning phase for example).

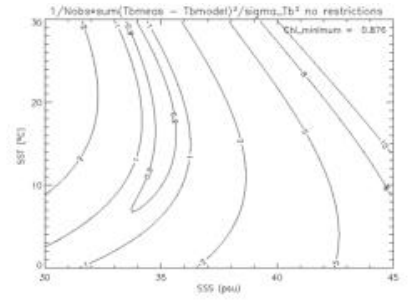

a)

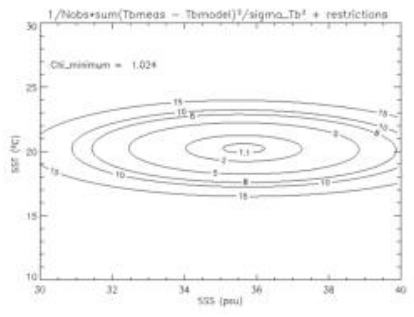

b)
Figure 2. Cost function contour plot when varying SSS and SST parameters without constraints (a) and with constraints (b).

Figure 2 shows the shape of the cost function when varying SSS and SST values. When no constraints are used the function does not have a clear minimum (plot a). The contour lines are almost vertical which means that $T_{B}$ is very little sensitive to SST changes (under-determination case), further confirming the need for a constrained cost function (plot $b$ ). Since the effect of having an error on SST of $0.5^{\circ}$ on the $T_{B}$ value is negligible in terms of SSS retrieval accuracy, the SST could alternatively be fixed (in the inversion) to measured or modeled values which are known with quite good precision (around $0.5^{\circ} \mathrm{C}$ ).

\section{ANALYSIS WITH SIMULATED SMOS IMAGES}

The three different cost functions have been also used in the inversion process to retrieve salinity from SMOS-like images using a SMOS simulator. The SMOS-like brightness temperature images have been generated by the SMOS Endto-end Performance Simulator (SEPS), and the SMOS Level 2 Processor has been used to perform the inversion. These tools are developed by the Universitat Politècnica de Catalunya [4], [5].

The simulations have been run using the combined Klein and Swift and Hollinger models and with the First Stokes Parameter configuration in the retrieval process. Also a $T_{B}$ bias cancellation technique has been performed as explained in [6], [7]. The true values (for SSS, SST, and WS) and the background uncertainties defined in section III are used together with the measurement $\left(T_{B}\right)$ uncertainties provided by SEPS. The optimization technique used to retrieve salinity is the Levenberg-Marquardt algorithm [8].

Figure 3 shows the histograms for the three different configurations, when no constraints are used (a), when constraints

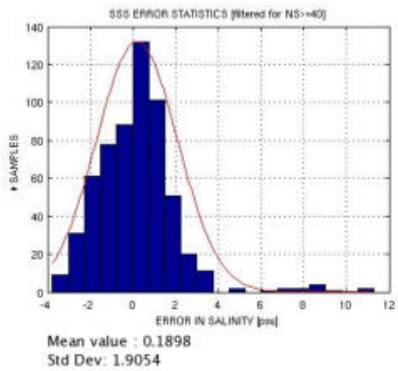

a1)

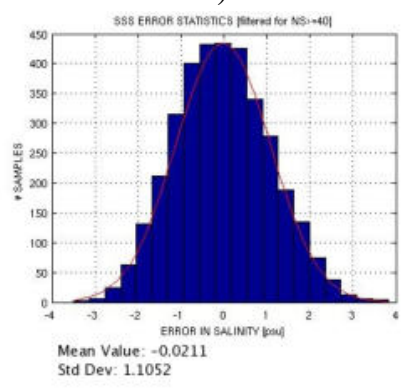

b1)

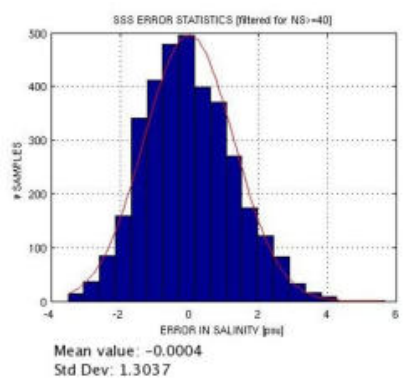

c1)

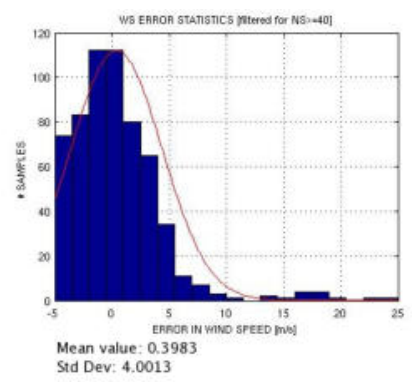

a2)

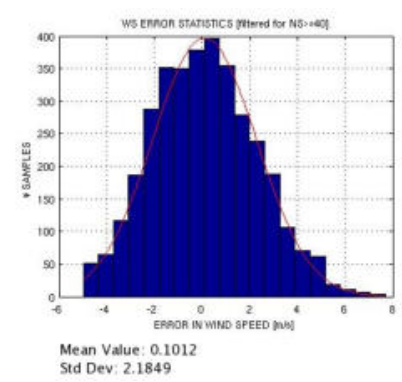

b2)

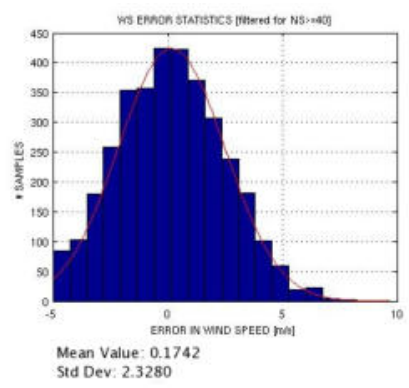

c2)
Figure 3. Histograms and statistics for the retrieved SSS values (1) and the retrieved WS values (2), for three different cases: when no constraints are included in the cost function (a), when all the constraints are considered (b) and when constraints are considered except for SSS parameter (c).

on all the parameters are considered (b) and when no restriction on SSS is considered (c).

Figure 3a exhibits a quasi Gaussian distribution but with large standard deviation, further confirming the already discussed effects of the low instrument sensitivities when no restrictions are considered. Note that the algorithm limits the range of possible solutions to an upper and a lower bound (due to the applicability range of the models) such that when the limit is reached, the inversion stops and no retrieval is performed. The acceptable ranges are $[0,30] \mathrm{m} / \mathrm{s}$ in WS, $[20,50]$ psu in SSS, and $[0,35]^{\circ}$ in SST. When no restrictions are considered, the processor rejects many points due to an out of bounds WS solution. As a consequence, the standard deviation of the distributions in case (a) is substantially smaller (about half) than if these out of bound cases would have been considered.

Comparing figures $3 \mathrm{~b}$ and $\mathrm{c}$, one can notice that the bias in the retrieved salinity are very similar, but the standard deviation is smaller for the case of using constraints for all 
parameters.

Several studies [9]-[11] have pointed out that if references on SSS are considered, then the retrieved SSS tends to the reference value, which is not the expected result. Figure 4 plots the difference between the retrieved and reference (or auxiliary) salinity (a) and also the difference between the retrieved and the original (truth) salinity values from the above simulations, for all the pixels of the field of view. It can be clearly appreciated that for the analyzed case, the retrieved salinity is not equal to the reference value. Moreover, the results in figures 3 and 4 show that more accurate retrieved salinities are obtained when the cost function is fully constrained (also with SSS), if this is done with realistic uncertainties.

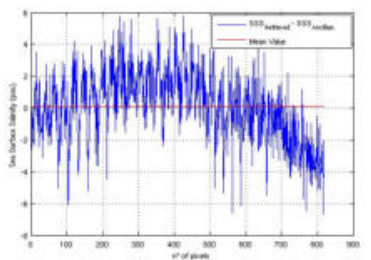

a)

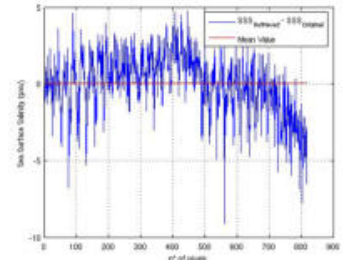

b)
Figure 4. Difference between retrieved and reference SSS (a) and between retrieved and original SSS (b) from the simulations results.

\section{Conclusions}

A sensitivity analysis is performed by looking at the shape of the different cost functions tested in this paper. When no constraints are considered, the minimum of the cost function is broad, which means that the sensitivity of $T_{B}$ to SSS and WS changes is very low. This indicates that to properly retrieve salinity constraints are necessary.

The very low sensitivity of $T_{B}$ to SST variations is manifested, so restrictions on SST are mandatory. Alternatively, SST can be fixed in the inversion process to a known (auxiliary) value.

It is essential to do a proper balancing between the observations and the background terms of the cost functions, to avoid that the retrieved parameter tends to the reference value.

From the SMOS simulations performed one can conclude that better results are obtained when all the constraints are considered. Also, from a theoretical point of view, there is no reason for not using the constraints on SSS, provided that the cost function is properly balanced and that all uncertainties are well characterized.

The authors therefore recommend to revisit the cost function algorithm as defined in the SMOS level 2 processor documentation (see ATBD description in [12]). More work should be done to fully characterize the SMOS inversion algorithm.

\section{ACKNOWLEDGMENT}

This study is a contribution to MIDAS-3 and MIDAS-4 projects funded by the Spanish National Program on Space (ESP2004-00671, ESP2005-06823-C05).

\section{REFERENCES}

[1] L. Klein and C. Swift, "An Improved Model for the Dielectric Constant of Sea Water at Microwave Frequencies," IEEE Transactions on Antennas and Propagation, vol. AP-25, no. 1, pp. 104-111, 1977.

[2] J. Hollinger, "Passive Microwave Measurements of Sea Surface Roughness," IEEE Transactions on Geoscience Electronics, vol. GE-9, no. 3, pp. 165-169, 1971.

[3] M. Portabella, "Wind field retrieval from stellite radar systems," Ph.D. dissertation, Universitat de Barcelona., 2002, iSBN: 90-6464-499-3.

[4] I. Corbella, A. Camps, M. Zapata, F. Marcos, F. Martínez, and Vallllossera, "End-to-end simulator of 2D interferometric radiometry," Radio Science, vol. 38, no. 8058, p. doi:10.1029/2002RS002665, 2003.

[5] A. Camps, I. Corbella, N. Vall-llossera, M.and Duffo, F. Marcos, Martínez-Fadrique, and M. Greiner, "The SMOS End-to-end Performance Simulator: Description and Scientific Applications," Proceedings International Geoscience and Remote Sensing Symposium, Toulouse, France, 2003.

[6] A. Camps, M. Vall-llossera, N. Duffo, and I. Corbella, "Retrieving Sea Surface Salinity with Multi-Angular L-band Brightness Temperature: Improvement by Spatio-Temporal Averaging," Radio Science, vol. 40, no. RS2003, doi:10.1029/2004RS003040, 2005

[7] M. Talone, A. Camps, R. Sabia, and J. Font, "Towards a Coherent Sea Surface Salinity Product from SMOS Radiometric Measurements and ARGO Buoys," Proceedings International Geoscience and Remote Sensing Symposium, Barcelona, 2007.

[8] W. Press, S. Teukolsky, W. Vetterling, and B. Flannery, Numerical Recipes in C. The Art of Scientific computing, 2nd edition. Cambridge University Press, 1992.

[9] "Synergetic Aspects and Auxiliary Data Concepts for Sea Surface Salinity Measurements from Space," ESA ESTEC, Tech. Rep., 2006, contract 18176/04/NL/CB Final Report.

[10] F. Petitcolin, J. Boutin, J. Vergely, P. Waldteufel, N. Reul, and J. Font, "Soil Moisture Retrieval for SMOS Mission, CCN2 for sea surface salinity retrieval,” European Space Agency, ESTEC, Tech. Rep., 2005, contract 16027/02/NL/GS, SMOS-TN-ACR-LOD-006. Final Report.

[11] R. Sabia, A. Camps, M. Vall-llossera, and N. Reul, "Impact on Sea Surface Salinity Retrieval of Different Auxiliary Data within the SMOS MIssion," IEEE Transactions on Geoscience and Remote Sensing, vol. 44, 2006

[12] J. Font, J. Boutin, N. Reul, P. Waldteufel, C. Gabarró, S. Zine, J. Tenerelli, F. Petitcolin, and J. Vergely, "SMOS Sea Surface Salinity Level 2 Algorithm Theoretical Baseline Document- Issue 2," European Space Agency, ESTEC, Tech. Rep., 2007, contract 18933/05/NL/FF. 